\title{
Channel length scaling and electrical characterization of graphene field effect transistor (GFET)
}

\author{
Reena Sri Selvarajan, Burhanuddin Yeop Majlis, Norliana Yusof, Azrul Azlan Hamzah \\ Institute of Microengineering and Nanoelectronics (IMEN), Universiti Kebangsaan Malaysia (UKM), Malaysia
}

\begin{tabular}{l}
\hline \hline Article Info \\
\hline Article history: \\
Received Oct 20, 2018 \\
Revised Feb 22, 2019 \\
Accepted Mar 5, 2019 \\
\hline
\end{tabular}

\section{Keywords:}

Ambipolar

Channel length

GFET

Short channel effect

Transfer characteristics

\begin{abstract}
The exclusive monoatomic framework of graphene makes it as an alluring material to be implemented in electronic devices. Thus, using graphene as charge carrying conducting channel material in Field Effect Transistors (FET) expedites the opportunities for production of ultrasensitive biosensors for future device applications. However, performance of GFET is influenced by various parameters, particularly by the length of conducting channel. Therefore, in this study we have investigated channel length scaling in performance of graphene field effect transistor (GFET) via simulation technique using Lumerical DEVICE software. The performance was analyzed based on electrical characterization of GFET with long and short conducting channels. It proves that conducting channel lengths have vast effect on ambipolar curve where short channel induces asymmetry in transfer characteristics curve where the n-branch is suppressed. Whereas for output characteristics, the performance of GFET heavily degraded as the channel length is reduced in short channels of GFET. Therefore, channel length scaling is a vital parameter in determining the performance of GFET in various fields, particularly in biosensing applications for ultrasensitive detection.
\end{abstract}

Copyright $(2019$ Institute of Advanced Engineering and Science. All rights reserved.

Corresponding Author:

Azrul Azlan Hamzah,

Institute of Microengineering and Nanoelectronics (IMEN),

Universiti Kebangsaan Malaysia (UKM),

Bangi, Malaysia.

Email: azlanhamzah@ukm.edu.my

\section{INTRODUCTION}

Graphene is a allotrope of carbon and two dimensional (2-D) material which is made up of single layer of carbon atoms organized in honeycomb lattice arrangement [1]. This exclusive monoatomic architecture of graphene offers pre-eminent properties such as high conductivity, high mechanical strength including $1 \mathrm{TPa}$ Young's Modulus and $130 \mathrm{GPa}$ tensile strength for single layer [2], high carrier mobilities up to which is 2-3 orders of magnitude higher than conventional semiconductor such as silicon. In addition, graphene is extremely sensitive to electric field and its neighboring charges as every atoms of it is disclosed to the surrounding. Thus, this give rise to making it as a favourable material to be applied in electronic devices, particulary for biosensing applications.

Graphene provides significant advantages over current standards in biosensing due to its unique properties which are ultrahigh sensitivity and excellent stability [3]. Among myriad electrical biosensing reported, devices based on field effect transistors have attracted much consideration [4]-[8]. A typical planar field effect transistor (FET) is consists of three contact conducting electrodes which are source(S), drain (D), and gate $(\mathrm{G})$ electrodes, thin insulating layer (dielectric) and a semiconducting layer where charge carriers flow. The current carrying channel is in direct contact with surrounding and this provides better control on surface charge [9]. Therefore, GFET biosensors are favorable and more sensitive as it able to directly translate interactions of biomolecules on its surface into readable electrical signals [10]-[12]. 
Hence, device parameters especially conducting channel length plays vital role in determining the effective performance of GFET for biosensing applications. Therefore, in this study we have investigated and reported significant effect of long and short channels for effective performance of GFET in future device applications.

\section{THEORY AND DESIGN}

\subsection{Theory}

Graphene offers unique electronic structure in which charge carriers (e.g. holes and electrons) obey linear energy band dispersion and its quasiparticles behave like massless Dirac fermions [13]. In single layer graphene (SLG) sheet, the Fermi level is located in between valence and conduction bands. Based on the band-structure calculations it shows that energy level in electronic structure of single graphene plane is not symmetrical. Asymmetry of electron and hole distribution causes shifting of the localized energy above or below Fermi level [14]. Thus, this allows graphene to exhibit strong ambipolar electric field effect such that charge carriers can be adjusted up between holes and electrons at room temperature by applying gate voltage [15] (Figure 1).

Therefore, the use of graphene as channel material in GFET exhibits unique electrical characteristics. This can be witnessed by transfer characteristics curve, typically known as ambipolar curve. The positive voltage offers n-type; while negative voltage demonstrates p-type conduction mechanism. The two branches of ambipolar curve is separated by Dirac point [16]. Experiment carried out by Novoselov. K.S.A illustrates that the conductivity of graphene doesn't goes zero and remains at finite a finite value of $\sim 4$ $\mathrm{e}^{2} / \mathrm{h}$ [17] although charge carriers depleted at the Dirac point [18]. However, the value of Dirac point voltage is dependent on some factors, such as: material used in making contact pads [19], quality of substrate used, graphene layer, density of charges at the interface of top and bottom of channel, and doping of the graphene sheet [20]. Hence, the performance of GFET can be observed and analyzed by its electrical characteristics.

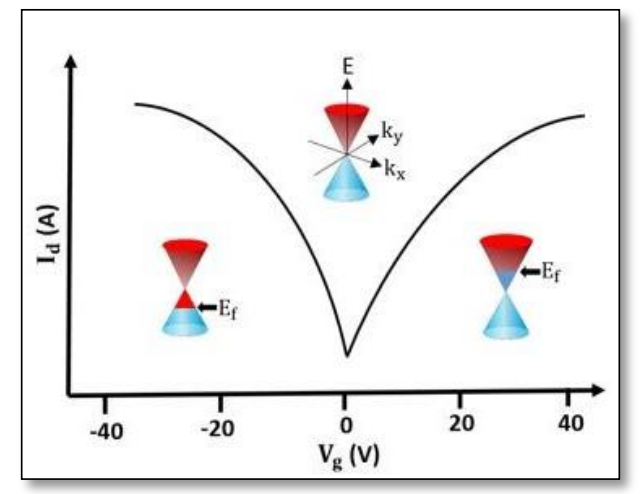

Figure 1. Schematic diagram of the band structure and ambipolar. The inset shows it's conical level low spectrum $\mathrm{E}(k)$, indicating changes in the position of the Fermi energy $\mathrm{E}_{\mathrm{f}}[21]$

\subsection{Design}

In this work, GFET with back gated architecture was modelled and simulated using Lumerical DEVICE Charge Transport (CT) solver. Three GFETs with different channel length (the gap between source and drain electrodes) were simulated to investigate channel length scaling on performance of GFET. Performance of GFET was studied and analyzed by its electrical characteristics. Long channel (4 um) and short channels $(600 \mathrm{~nm}$ and $100 \mathrm{~nm})$ were simulated respectively. Figure 2(a) represents the schematic diagram of GFET with back gate design. The device was simulated using (DEVICE CT) Lumerical DEVICE Charge Transport Solver as illustrated in Figure 2(b). 


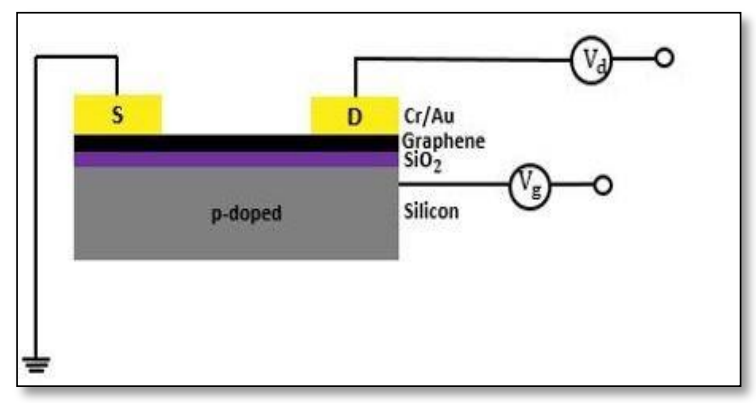

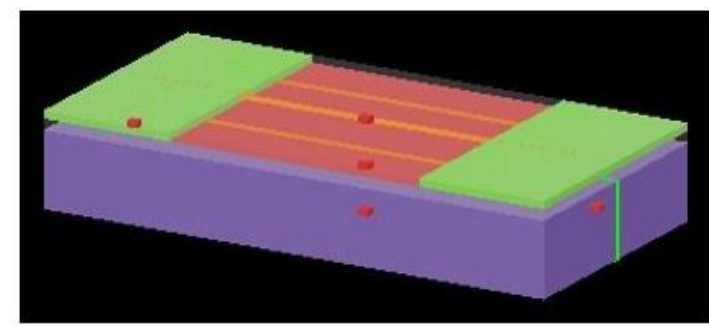

(b)

(a)

Figure 2. (a) Schematic diagram of back gate configuration design of GFET (b) Simulated model of backgated GEFT

GFET device with three different graphene channel length was simulated in DEVICE CT. Single layer graphene (SLG) with carrier mobility of $6000 \mathrm{~cm}^{2} / \mathrm{Vs}$ for both holes and electrons was set at initial temperature of $300 \mathrm{~K}$. Silicon wafer with $300 \mathrm{~nm}$ thickness of $\mathrm{SiO}_{2}$ was used as substrate for graphene deposition. Gold electrodes were used as source and drain electrodes respectively. This is because gold offers exclusive material properties compared to silver and platinum. The elementary reason is because gold is relatively inert and gives ideal metal contact [22]. Source electrode was grounded while highly p-doped silicon substrate was used as back gate electrode.

\section{RESULTS AND ANALYSIS}

The back gate GFET with three different channel lengths $(100 \mathrm{~nm}, 600 \mathrm{~nm}$, and $4 \mathrm{um})$ were simulated using Lumerical software to study its electrical characteristics transfer characteristics and output characteristics. Channel length dependent transport behavior was observed and reported in the section below.

\subsection{Transfer Characteristics Curve}

Graph of drain current $I_{d}$ as a function of gate voltage $\mathrm{Vg}$ at low drain bias $\left(\mathrm{V}_{\mathrm{d}}=0.1 \mathrm{~V}\right.$ and $\left.0.3 \mathrm{~V}\right)$ for three different channel lengths are shown in Figure 3.

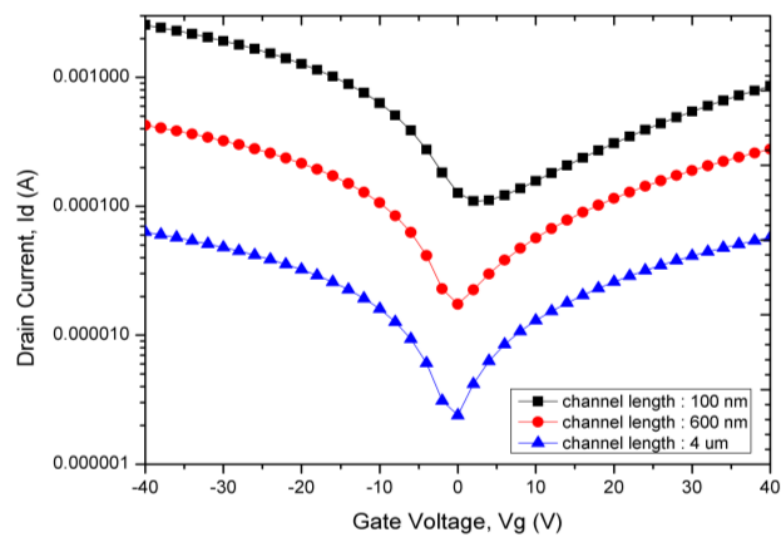

Figure 3. Transfer characteristics curve (ambipolar curve) of GFET, $\mathrm{I}_{d} \mathrm{vs} \mathrm{Vg}$ for GFET with three different channel length: $100 \mathrm{~nm}, 600 \mathrm{~nm}$ and $4 \mathrm{um}$

Figure 3 shows transfer characteristics curve of GFET that exhibits ambipolar characteristics which proves the unique electronic ability of single layer graphene to operate in both p-type and n-type conduction regions. The Dirac point, commonly known as charge neutrality point occurs at $\mathrm{Vg}=0 \mathrm{~V}$ and the drain current remain at a finite value for all the three geometries of GFET. This indicates that at this point, GFET exhibits highest resistivity and minimum conductivity. Researchers have reported that through DC 
measurement, GFET experiences minimum conductivity in the order of $4 \mathrm{e}^{2} / h$. The position of Dirac point in the transfer characteristics curve is an indicator to monitor the doping state of the graphene layer.

It is worthy to take note that asymmetry between $\mathrm{n}$ - and $\mathrm{p}$ - branches becomes more distinct for short-channel devices compared to long channel. Figure 3 illustrates that the GFET with channel length of $100 \mathrm{~nm}$ exhibits asymmetry in ambipolar curve where the $\mathrm{n}$-branch is suppressed. On the other hand, GFET with 4um channel length shows symmetrical distribution of ambipolar curve in its both $\mathrm{p}$ - and $\mathrm{n}$-type branches [23]. This proves that length of conducting channel in GFET devices influence its transport behavior. Therefore, as the channel length reduces, the asymmetry between the ambipolar curve become more conspicuous.

For long channels especially in 4um channel length of GFET, the transport of charge carriers are dominated by graphene channel. However, as the channel length is reduced it's widely controlled by the interaction between the graphene and metal contacts. In short channel devices, graphene underneath metal contacts can be doped either to be p- or n-type depending on the work function of metal contacts, charges transfer from metal contact to graphene, and depending on the polarity of carriers in graphene. Thus, this in turn leads to formation of p-p or p-n junction in graphene [24].

In this simulation, work function mismatch between graphene and the metal contacts in short channels, p-doped the graphene layer. Therefore, as the channel is shifted by the gate to $n$-region, p-n junction is formed between source/drain contacts and graphene. Hence, this introduces resistance in the conducting channel and limits the current injection. n-regime of ambipolar branch is suppressed in short channel as shown in curve of channel length 100nm. Therefore, transport in short channel of GFET is dominated by interaction between and graphene and metal contacts which may affect the transport of charge carriers in the device. Hence, this in turn affects the performance of GFET.

\subsection{Output Characteristics}

Figure 4 illustrates the output characteristics of GFET with three different channel length such that drain current $I_{d}$ as a function of drain voltage $V_{d}$ for various gate voltage $V_{g}$. The output characteristics curve of the simulated GFET reported in Figure 4(a) are similar to previous demonstrations at these channel length [25]. However, for short channels such as $100 \mathrm{~nm}$ and $600 \mathrm{~nm}$, the output characteristics curve exhibits different properties. The discrepancy of lines in the curve become less distinctive as the gate voltage varied from $0.5 \mathrm{~V}$ to $2.0 \mathrm{~V}$. Drain current for the channel tends to become closer values for wide range of gate voltage applied (Figure 4(b)). Especially for channel length with $100 \mathrm{~nm}$, the device exhibits same output characteristics for all gate biases ranging from $0.5 \mathrm{~V}$ to $2.0 \mathrm{~V}$. All the drain current values converged into one curve and exhibits same values (Figure 4 (c)) although gate voltages are varied from $0.5 \mathrm{~V}$ to $2.0 \mathrm{~V}$.

In comparative with long channel devices (Figure 4(a)), it is worthy to take note that this systematic degradation of drain current can be witnessed with decreasing channel length [Figure 4(b) and 4(c)]. Although, Figure 4(c) demonstrates highest drain current magnitude compared to GFET with 4 um and 600 $\mathrm{nm}$ respectively, however degradation in output characteristics curve affects its performance. The device unable to show difference in drain current values as different gate bias is applied. This is due to trap charges in the oxide or graphene oxide interface affects the transport of charge carriers in GFET. Eventually, high drain-to-source biasing has stronger impact on short channels as it creates hot carrier injection. Therefore, short channels are sensitive and can be influenced by trap-charges which in turn degrades the performance of GFET.

\subsection{Effect of Circuit Temperature on Performance of GFET}

Figure 5 shows performance of GFET in various circuit temperature ranging from $173 \mathrm{~K}$ to $373 \mathrm{~K}$. Electrical transport of charge carriers through graphene channel exhibits compelling changes as the temperature alternate above and below room temperature. Increasing temperature above room temperature, shows drastic increase in the magnitude of drain current, $\mathrm{I}_{d}$ of the device. This proves that the maximum resistance in the device is reduced as the temperature increases and aids in the effective performance of GFET. However, it's also worthy to take note that decreasing simulation temperature below room temperature, reduces the performance of GFET. The magnitude of drain current reduces and n-branch of ambipolar curve is suppressed as temperature reduces. On the other hand, this demonstrates that there is significant increase in maximum resistance in the GFET device. Therefore, varying simulation temperature influences the transport of charge carriers via graphene channel in GFET, in which affects the performance of the device. 


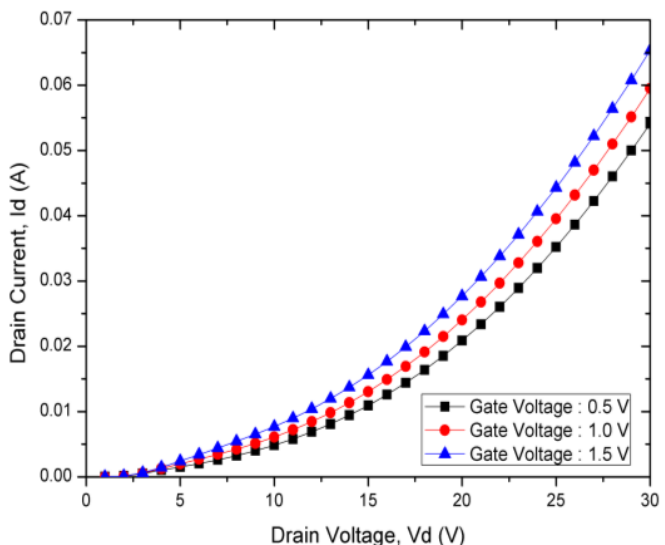

(a)

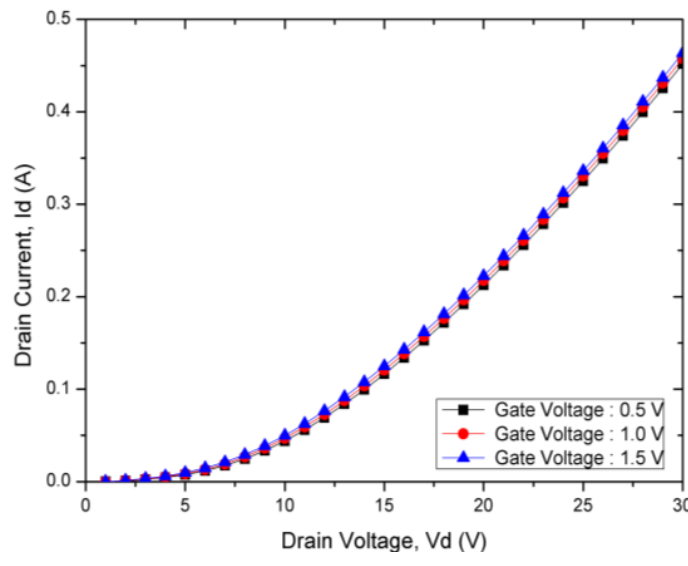

(b)

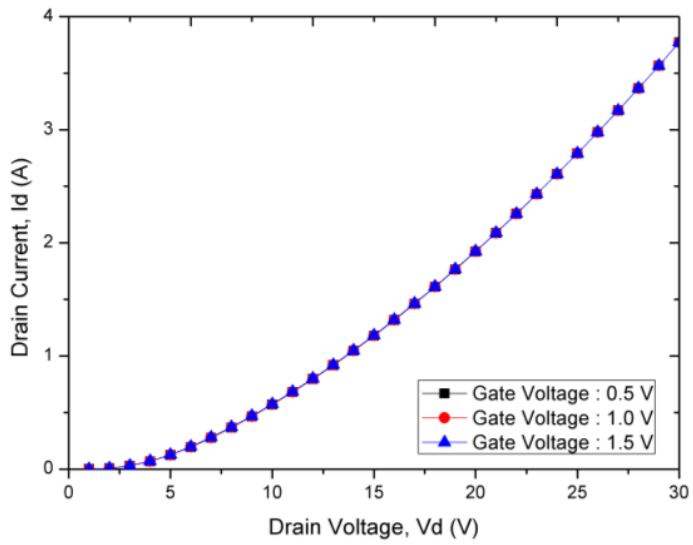

(c)

Figure 4. Output characteristics curve of GFET, $\mathrm{I}_{\mathrm{d}} \mathrm{Vs} V_{d}$ at $\mathrm{Vg}$ ranging from 0 to $90 \mathrm{~V}$. (a) 4 um channel length, (b) $600 \mathrm{~nm}$ channel length, and (c) $100 \mathrm{~nm}$ channel length

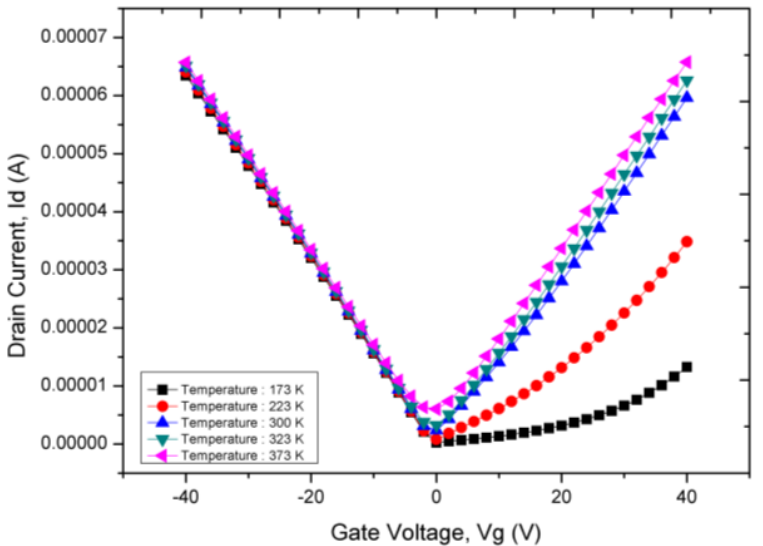

Figure 5. Temperature dependence transport of charge carriers in GFET

\section{CONCLUSION}

In conclusion, this paper highlights the importance of channel length scaling in effective performance of graphene based field effect transistor (GFET). Proposed GFET model was simulated in Lumerical DEVICE charge solver (DEVICE CT) software and electrical characteristics of the device were 
analyzed. Long channel which is 4 um exhibits symmetrical ambipolar curve, whereas short channel devices (e.g. $100 \mathrm{~nm}$ and $600 \mathrm{~nm}$ ) demonstrates an asymmetry pattern in the ambipolar curve, where the $\mathrm{n}$-branch is suppressed. In addition, for output characteristics, the performance of GFET heavily degraded as the channel length is reduced. It is also worthy to take note that, simulation temperature has significant impact on the performance of the device. Therefore, surrounding temperature needed special attention for effective transport of charge carriers in the GFET device. Hence, channel length scaling is a vital parameter in determining the performance of GFET in various fields, particularly in biosensing applications. In conclusion, long channel length should be implemented in fabrication of GFET to ensure effective performance of the device.

\section{ACKNOWLEDGEMENTS}

This research is supported by AKU-95 HICoE MEMS for Artificial Kidney grant funded by the Ministry of Higher Education (MOHE) Malaysia.

\section{REFERENCES}

[1] F. Yan, M. Zhang, and J. Li, "Solution-Gated Graphene Transistors for Chemical and Biological Sensors," Adv. Healthc. Mater., vol. 3, no. 3, pp. 313-331, 2014.

[2] J. Hwang et al., "Enhanced mechanical properties of graphene/copper nanocomposites using a molecular-level mixing process," Adv. Mater., vol. 25, no. 46, pp. 6724-6729, 2013.

[3] Afsahi, S., Lerner, M. B., Goldstein, J. M., Lee, J., Tang, X., Bagarozzi, D. A., Goldsmith, B. R. (2018). Novel graphene-based biosensor for early detection of Zika virus infection. Biosensors and Bioelectronics, 100(August 2017), 85-88.

[4] Haslam, C., Damiati, S., Whitley, T., Davey, P., Ifeachor, E., \& Awan, S. (2018). Label-Free Sensors Based on Graphene Field-Effect Transistors for the Detection of Human Chorionic Gonadotropin Cancer Risk Biomarker. Diagnostics, 8(1), 5.

[5] Marsi, N., Majlis, B. Y., \& Hamzah, A. A. (2014). The Mechanical and Electrical Effects of MEMS Capacitive Pressure Sensor Based 3C-SiC for Extreme Temperature The Mechanical and Electrical Effects of MEMS Capacitive Pressure Sensor Based 3C-SiC for Extreme Temperature, 2014(May).

[6] Marsi, N., Majlis, B. Y., Mohd-Yasin, F., \& Hamzah, A. A. (2015). The fabrication of back etching 3C-SiC-on-Si diaphragm employing $\mathrm{KOH}+$ IPA in MEMS capacitive pressure sensor. Microsystem Technologies, 21(8), 1651-1661.

[7] Selvarajan, R. S., Hamzah, A. A., \& Majlis, B. Y. (2017). Transfer Characteristics of Graphene based Field Effect Transistor (GFET) for Biosensing Application, 88-91.

[8] Hamzah, A. A., Yunas, J., Majlis, B. Y., \& Ahmad, I. (2008). Sputtered encapsulation as wafer level packaging for isolatable MEMS devices: A technique demonstrated on a capacitive accelerometer. Sensors, 8(11), 7438-7452.

[9] R. Chaudhary et al., "Fabrication and characterisation of Al gate n-metal-oxide-semiconductor field-effect transistor, on-chip fabricated with silicon nitride ion-sensitive field-effect transistor," IET Comput. Digit. Tech., vol. 10, no. 5, pp. 268-272, 2016.

[10] Y. Cui, "Nanowire Nanosensors for Highly Sensitive and Selective Detection of Biological and Chemical Species," Science (80-. )., vol. 293, no. 5533, pp. 1289-1292, 2013.

[11] Haslam, C., Damiati, S., Whitley, T., Davey, P., Ifeachor, E., \& Awan, S. (2018). Label-Free Sensors Based on Graphene Field-Effect Transistors for the Detection of Human Chorionic Gonadotropin Cancer Risk Biomarker. Diagnostics, 8(1), 5. [7]Liang, J., Kohsaka, F., Matsuo, T., Li, X., \& Ueda, T. (2008). Improved bi-layer lift-off process for MEMS applications. Microelectronic Engineering, 85(5-6), 1000-1003.

[12] Ordonez, R. C., Hayashi, C. K., Torres, C. M., Melcher, J. L., Kamin, N., Severa, G., \& Garmire, D. (2017). Rapid Fabrication of Graphene Field-Effect Transistors with Liquid-metal Interconnects and Electrolytic Gate Dielectric Made of Honey. Scientific Reports, 7(1), 1-9. https://doi.org/10.1038/s41598-017-10043-4

[13] K. Xu, J. Qian, P. Shukla, M. Dutta, and M. a. Stroscio, "Graphene-based FET structure: Modeling FET characteristics for an aptamer-based analyte sensor," 2012 15th Int. Work. Comput. Electron. IWCE 2012, 2012.

[14] X. Ma, W. Gu, J. Shen, and Y. Tang, "Investigation of electronic properties of graphene/Si field-effect transistor.," Nanoscale Res. Lett., vol. 7, no. 1, p. 677, 2012.

[15] D. Chen, L. Tang, and J. Li, "Graphene-based materials in electrochemistry," Chem. Soc. Rev., vol. 39, no. 8, pp. 3157-3180, 2010.

[16] Fu, W., Feng, L., Panaitov, G., Kireev, D., Mayer, D., Offenhäusser, A., \& Krause, H. J. (2017). Biosensing near the neutrality point of graphene. Science Advances, 3(10).

[17] P. Sharma, J. S. Gomez-Diaz, A. M. Ionescu, and J. Perruisseau-Carrier, "Determination of minimum conductivity of graphene from contactless microwaves measurements," Proc. IEEE Conf. Nanotechnol., 2012.

[18] A K. Geim and K. S. Novoselov, “The rise of graphene," Nat. Mater., vol. 6, no. 3, pp. 183-191, 2007.

[19] Liang, C., Wang, Y., \& Li, T. (2016). Studies on contact resistance in graphene based devices. Microsystem Technologies, 22(8), 1943-1947. 
[20] M. Luszczek, M. Turzyński, and D. Świsulski, "Modelling of Graphene Field-Effect Transistor for electronic sensing applications," Przeglad Elektrotechniczny, no. 10, pp. 170-172, 2015.

[21] R. Sri, A. Azlan, and B. Yeop, "Electrical Characteristics of Graphene based Field Effect Transistor ( GFET ) Biosensor for ADH Detection."

[22] A. Manuscript and S. P. C. Nanostructures, "NIH Public Access," Nano, vol. 6, no. 9, pp. 2166-2171, 2008.

[23] G. F. Transistors, Z. Chen, S. Han, Z. Chen, A. A. Bol, and Y. Sun, "Channel-Length-Dependent Transport Channel-Length-Dependent Transport Behaviors of Graphene Field-Effect Transistors,” no. July, 2011.

[24] I. Meric et al., "Channel Length Scaling in Graphene Field-Effect Transistors," 2011.

[25] D. Chen, L. Tang, and J. Li, "Graphene-based materials in electrochemistry." Chem. Soc. Rev., vol. 39, no. 8, pp. 3157-80, 2010 .

\section{BIOGRAPHIES OF AUTHORS}

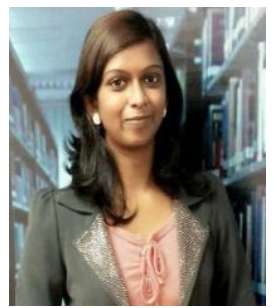

Reena Sri Selvarajan received B.Eng (Hons.) from Universiti Malaysia Perlis (UniMAP), majoring in biomedical electronics engineering. She is currently pursuing her $\mathrm{PhD}$ in Micro and Nanoelectronics engineering in Institute of Microengineering and Nanoelectronics (IMEN), Universiti Kebangsaan Malaysia, 43600 Bangi, Selangor, Malaysia. Her research nterest includes include Micro-Electro-Mechanical Systems (MEMS) devices and biosensors.

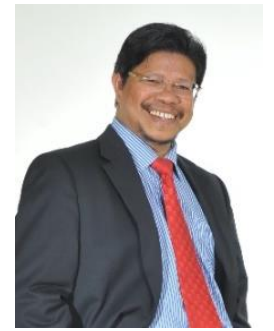

Burhanuddin Yeop Majlis is a professor of microelectronics at the Faculty of Engineering. He received his Ph.D. in microelectronics from University of Durham, United Kingdom in 1988, MSc in microelectronics from University of Wales, UK in 1980 and BSc(Hons.) in Physics from UKM in 1979. He was a Deputy Dean of Engineering Faculty from 1995 until 1997. He is also a Research Fellow of Telekom Malaysia Research \& Development Division, and he was the director of UKM-TM Microelectronics Research Centre at the Faculty of Engineering, UKM. He was responsible in developing and planning the setting up of the clean room for research at UKM. He had attended intensive industrial training in GaAs MMIC design and manufacture at GEC-Marconi Material Technology Ltd. United Kingdom. He is a senior member of the Institution of Electrical and Electronics Engineer (IEEE) and the Chairman of IEEE Electron Devices Malaysia Chapter from 1994 to 2006. He also Fellow Member of Malaysian Solid State Science and Technology Society (FMASS). He is the founder chaiman and President of Malaysia Nanotechnology Association (MNA) which is established in 2007. He initiated research in microfabrication and microsensors at UKM in 1995 and has also initiated research in GaAs technology with Telekom Malaysia. In 2001 he stared research in MEMS with substantial research funding of US\$10 million from Ministry of Science, Technology and Innovation. His current interest are design and fabrication of MEMS sensor, RFMEMS, BiOMEMS and microenergy. He has published four text books in electronics and one book on Integrated Circuits Fabrication Technology for undergraduate courses and more than 400 academic research papers. Now he is the founder director of Institute of Microengineering and Nanoelectronics (IMEN).

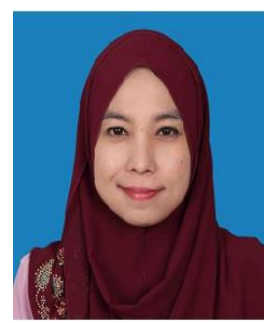

Norliana Yusof received the B.Eng (Hons.) from Universiti Teknologi Malaysia and the M.Eng degree from University of Malaya, Malaysia, in 2004 and 2010, respectively. She is currently a $\mathrm{PhD}$ student in Institute of Microengineering and Nanoelectronics (IMEN), Universiti Kebangsaan Malaysia, 43600 Bangi, Selangor, Malaysia. Her current research interests include Micro-Electro-Mechanical Systems (MEMS) devices.

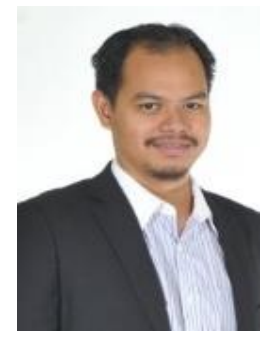

Azrul Azlan Hamzah is the director of Institute of Microengineering and Nanoelectronics (IMEN), UKM. He receieved his $\mathrm{PhD}$ in microelectronics from IMEN, UKM in 2008 and BSc in manufacturing engineering from University of California in 2000. His current interest are design and fabrication of MEMS sensor, RFMEMS, BiOMEMS and microenergy. 